\title{
OUTLIERS DETECTION BY RANSAC ALGORITHM IN THE TRANSFORMATION OF 2D COORDINATE FRAMES
}

Detecção de valores aberrantes com o uso de algoritmo RANSAC na transformação de coordenadas rectangulares.

\author{
JOANNA JANICKA \\ JACEK RAPINSKI
}

University of Warmia and Mazury in Olsztyn, Institute of Geodesy

ul. Oczapowskiego 1, 10-719 Olsztyn

joannasuwm@gmail.com; jacek.rapinski@uwm.edu.pl

\begin{abstract}
Over the years there have been a number of different computational methods that allow for the identification of outliers. Methods for robust estimation are known in the set of M-estimates methods (derived from the method of Maximum Likelihood Estimation) or in the set of R-estimation methods (robust estimation based on the application of some rank test). There are also algorithms that are not classified in any of these groups but these methods are also resistant to gross errors, for example, in M-split estimation. Another proposal, which can be used to detect outliers in the process of transformation of coordinates, where the coordinates of some points may be affected by gross errors, can be a method called RANSAC algorithm (Random Sample and Consensus). The authors present a study that was performed in the process of $2 \mathrm{D}$ transformation parameter estimation using RANSAC algorithm to detect points that have coordinates with outliers. The calculations were performed in three scenarios on the real geodetic network. Selected coordinates were burdened with simulated values of errors to confirm the efficiency of the proposed method.
\end{abstract}

Keywords: Coordinate Transformation; RANSAC; Parameter Estimation.

\section{RESUMO}

Ao longo de anos elaboraram-se muitos métodos de cálculos que permitem a identificação de erros grosseiros nas observações. Foram desenvolvidos os métodos de estimação robusta no conjunto dos métodos definidos como M-estimações 
(derivados do método Maximum Likelyhood Estimation) ou R- estimações. Outros métodos são também conhecidos que não se incluem a nenhum desses grupos, e que também, mostram resistência aos erros grossos, por exemplo, Msplit estimação. A proposta neste artigo, que pode se aplicar ao processo de transformação de coordenadas, quando as coordenadas de alguns de pontos podem estar contaminadas por erros grosseiros, é o método denominado de RANSAC algoritmo (Random Sample and Consensus). Os autores apresentam a possibilidade da utilização deste método numérico para a detecção erros grosseiros nas coordenadas de pontos utilizados na estimação dos parâmetros de transformação. Experimentos foram realizados com três cenários de erros nos dados utilizando uma rede real de pontos. Os resultados obtidos nos experimentos realizados com dados simulados foram animadores e confirmam a eficiência do algoritmo proposto grosseiros para verificar a eficácia do método proposto.

Palavras-chave: Transformação de Coordenadas, RANSAC, Estimação de Parâmetros.

\section{INTRODUCTION}

The transformation of coordinates allows for the conversion of coordinates from one geodetic system to another. Usually the determination of transformation parameters is performed by the means of the least-squares method. Unfortunately, the least squares method is not immune to outliers. In geodetic practice, each observation is measured with an unknown true error. Among those errors there are: systematic errors, random errors and gross errors. Observations burdened with gross errors are called outliers, thus outliers are sample values that are significantly different from the rest of the sample (GRUBBS, 1969). In coordinate transformation there are also situations when the coordinates of some reference points are burdened with gross errors. The outliers should be identified and eliminated from the process of transformation parameter estimation. One can also decrease their influence on the estimated transformation parameters by applying special estimation methods e.g.: robust M-estimation (maximum likelihood type estimator) (HUBER, 1964; JANICKA, 2011), R-estimation (DUCHNOWSKI, 2011) or the M-split estimation (WSNIEWSKI, 2008) (estimation which results in two or more resulting group of competing parameters). The application of robust estimation methods in the process of coordinate transformation has been tested. The obtained results confirmed the effectiveness of these methods (JANICKA \& RAPINSKI, 2013). The biggest problem occurs when the dataset has many points with gross errors in their coordinates. If the number of outliers is greater than $40-45 \%$, these methods do not generate the correct results. Except from strictly statistical methods, there is a group of numerical methods, which deal with outliers (ISACK and BOYKOV, 2012; CHOI et. al., 2009). In literature the RANSAC algorithm is often mentioned as a good method to deal with such a data set. Therefore, in this paper, it is proposed to apply a RANSAC method (FISCHLER \& BOLLES, 1981). In the next section, the 
general RANSAC algorithm is described. Section 3 presents implementation of this algorithm for coordinate transformation. Section 4 shows the results of tests of the above procedure using a data set with various numbers of outliers.

\section{2 . RANSAC ALGORITHM}

The RANSAC algorithm was first introduced by Fischler and Bolles in 1981 as a method to estimate the parameters of a certain model, starting from a set of data contaminated by large amounts of outliers. It is an iterative, non-deterministic algorithm which uses least-squares to estimate model parameters. The basic premise of RANSAC is the presence in the data set of both observations that fit the model (inliers) and those which differ from the values (outliers). The sources of data that do not fit into the model are gross errors (measurement errors), noise or other disturbances. The input data of the algorithm are: a set of data and a mathematical model that will be matched to the data set. The advantage of this method is that the percentage of outliers which can be handed by RANSAC can be larger than $50 \%$ of the entire data set (MURRAY \& TORR, 1997). Such a percentage, known also as the "breakdown points", is commonly assumed to be the practical limit for many other commonly-used techniques for parameter estimation (such as a robust estimation method, for example, for the Huber, Hampel and Danish methods).

The RANSAC algorithm is essentially composed of two steps that are repeated in an iterative process:

1. Hypothesis,

2. Tests.

Apriori information, which is used in the process of fitting the model includes:

1. Minimum number of points (observations) required to fit the model;

2. Minimum number of iterations;

3. Parameter $\left(\mathrm{m}_{\mathrm{d}}\right)$ determining the threshold that splits the inliers from outliers in the process of hypothetical model testing;

4. The size of the data set, which completes the process of the iteration.

The hypothesis step is related to the concept of the minimal sample set (MSS). The first minimal sample set is randomly selected from the input dataset and the model parameters are computed using only the elements of the MSS. The MSS is the amount of data (observations) required to compute the parameters of the model (the minimum number of observations that is required to describe the model is equal to the number of model parameters). The minimum number is determined by the selected function describing the model. For the 2D Helmert transformation, the minimum number of points is 2 with $2 \mathrm{D}$ coordinates in both frames. Thus, the first phase starts with selecting a necessary and minimum number of observations of the data set. Based on these selected observations, the output model (hypothetical) is estimated. All of the remaining data are tested in terms of fit to the hypothetical model. If one or both of selected points are burdened with outliers, then hypothetical model won't fit the rest of data. Therefore the algorithm will skip this model and randomly pick another pair of points for another hypothetical model. 
In the testing step, RANSAC iteratively checks which observations of the entire dataset are consistent with the hypothetical model. This requires determining the value of the parameter $m_{d}$ specifying the maximum distance from a test point to a hypothetical model. If it fits the criterion of $m_{d}$, the point is treated as just another hypothetical inlier. The minimum percentage of observations that must be the correct data in the whole data set must be also defined by user (for example, the model can be regarded as properly defined if $80 \%$ of the observations are those that are not burdened with outliers). The estimated model is correct if it has a sufficient number of points that have been classified as correct observations (inliers). The best set of observations which is selected from the entire dataset is called the consensus set (CS). Defining iteration as a single process of random selection of MSS and fit testing, the number of iterations is determined by the following formula:

$$
T_{i t e r}=\frac{\log \mathcal{E}}{\log (1-q)}
$$

where:

$\varepsilon$ - probability of incorrect identification of the model,

$q-$ is calculated based on the following equation:

$$
\mathrm{q}=\left(\mathrm{N}_{\mathrm{i}} / \mathrm{N}\right)^{\mathrm{k}}
$$

in which: $N_{i}$ - the number of points that belong to the consensus set, $N-$ the total number of points, $k$ - minimal number of data that are necessary to clearly define the model.

If we want to obtain an error-free selection of points (MSS) with a probability $1-\varepsilon$, we need to perform at least $\mathrm{T}$ iterations.

Figure 1 depicts an example of the application of RANSAC to estimate line parameters. The data set containing correct observations (inliers) and observations burdened with gross errors (outliers) is shown in Figure 1.

Figure 1 - The data set observations.

\begin{tabular}{|c|c|}
\hline $\begin{array}{c}\text { Figure 1(a) - A data set with many } \\
\text { outliers. }\end{array}$ & $\begin{array}{c}\text { Figure 1(b) - Line fitted with RANSAC } \\
\text { which a line has to be fitted }\end{array}$ \\
\hline & \\
\hline
\end{tabular}

Bol. Ciênc. Geod., sec. Artigos, Curitiba, v. 20, nº 3, p.610-625, jul-set, 2014. 
Figure 1(a) presents the entire input data set, from which the correct observations describing a straight line are selected. Figure 1(b) shows the line that is based on the correct observation (inliers) selected by the RANSAC algorithm and the solid dots are the outliers that do not fit the model.

\section{RANSAC APPLICATION IN TRANSFORMATION PARAMETER ESTIMATION}

The RANSAC algorithm described in the previous section can be used in transformation parameter estimation. It is particularly recommended if the results of standard least squares estimation are not satisfactory. It can imply that outliers are present in the set of reference points. To confirm the effectiveness of the proposed method, the Helmert transformation was adopted. Traditionally, determination of transformation parameters is performed by the least-squares method using all available points in a single computational process. Unfortunately, since the least squares method is not a robust method, this method is not immune to outliers. It means that if, for any reason, one or more of the reference point coordinates is not correct, the transformation parameters calculated using least squares will be estimated with this error. Such observations must be eliminated from the data set before performing least square estimation of coordinate transformation parameters. In this paper, the RANSAC algorithm for estimation of coordinate transformation parameters is proposed to complete this task. The RANSAC algorithm used during the parameter estimation process allows selecting only those points that are not outliers to be used in the estimation. In each step of this algorithm, the transformation parameters are still estimated by the least squares method. The greatest benefit of this approach is that the percentage of outliers can be larger than $50 \%$ of the entire set of reference points.

The RANSAC transformation is an iterative process and is described as follows:

1. Helmert transformation in two-dimensional space is a four-parameter transformation. Thus, the minimum number of reference points is two. In the first step, two reference points are randomly selected from the entire set of reference points and the transformation parameters are calculated by solving a set of four linear equations. This is the step of creation of the hypothetical model.

2 . The next step is the transformation of reference points coordinate from one Cartesian coordinate system to another (e.g. old local Cartesian coordinate system to the national 2000 coordinate system used in Poland) with the hypothetical transformation parameters (from the first step, on the basis of the hypothetical model).

3. Definition of the parameter $m_{d}$, the minimum number of iterations and the minimum number of points (observations) required to fit the model. This is an arbitrary parameter which corresponds to the maximum allowable residual after adjustment, so the value of this parameter depends on the required accuracy of transformation.

Bol. Ciênc. Geod., sec. Artigos, Curitiba, v. 20, nº 3, p.610-625, jul-set, 2014. 
4. Calculation of Euclidean distances to each point from the points transformed using a hypothetical model and testing conditions: if $d<$ md then the point is added to the minimal set of reference points described in the first step, then if the number of inliers is sufficient (e.g. min $80 \%$ of the entire set of total points).

5. If all conditions from step 4 are satisfied, the iteration process is finished (Fig.2). The Consensus Set (CS) is then defined and based on it, the transformation parameters are re-estimated by the least-squares method. If conditions are not fulfilled, then RANSAC algorithm once again selects the minimum number of points to define a hypothetical model and the procedure 1-4 is repeated.

6 . The final step is the transformation of coordinates from one geodetic system to another using the CS model parameters.

Figure 2 presents the block diagram of the RANSAC transformation.

Figure 2 - Block diagram of RANSAC algorithm.

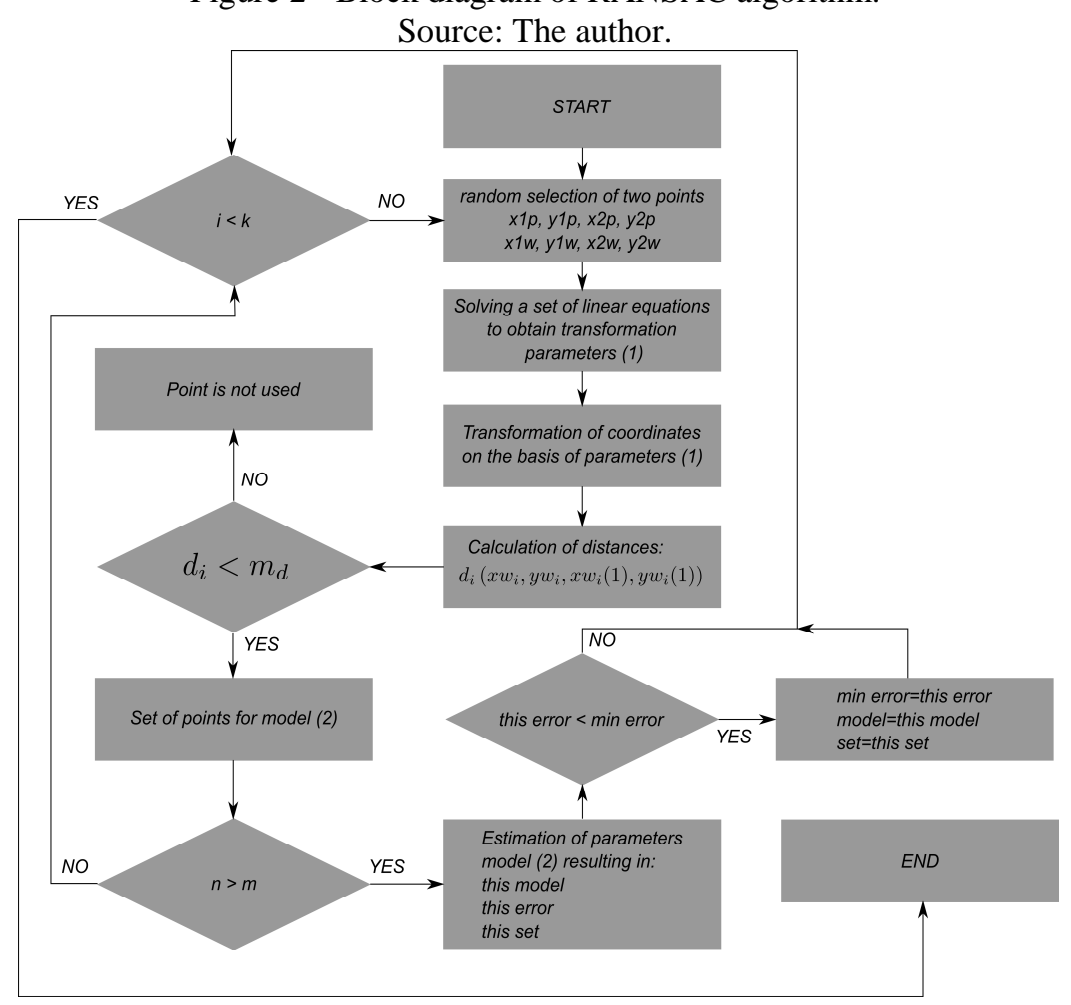

In the Figure 2, $i$ stands for the current iteration, $k$ is the maximum number of iterations, $n$ is a number of points selected in one iteration, $m$ is the minimum number of points for which the model is estimated, $x 1 p, y 1 p, x 2 p, y 2 p$ are 
coordinates in primary coordinate system, $\mathrm{x} 1 \mathrm{w}, \mathrm{y} 1 \mathrm{w}, \mathrm{x} 2 \mathrm{w}, \mathrm{y} 2 \mathrm{w}$ are coordinates in the secondary coordinate system.

\section{EXAMPLE}

An analysis performed using real data (i.e., a horizontal geodetic network) confirmed the effectiveness of the proposed algorithm. This object (named JAROCIN) is a horizontal geodetic network, which includes 158 points. The coordinates of these points are defined in the local coordinate system and in the Polish Coordinate System "2000", where 54 of them are reference points. The "2000" reference frame was introduced in Poland in 2000 as a new national reference frame after the ETRF (European Terrestrial Reference Frame) was adopted. Since the previous reference frame in Poland was based on the Krassowski ellipsoid, it was necessary to define a new system and create a new database of control networks in the "2000" reference frame. There are two ways in which this task can be accomplished: re-surveying of the entire networks and new adjustment in the 2000 frame and transformation of coordinates from the previous reference frames (either "1965" or local).

Re-surveying is preferable in the case of the high order networks. Transformation was allowed in the case of local coordinate frames and lower order networks. In many cases, a problem with outliers in the coordinates of reference points occurred in the transformation process. Reference points (points used to calculate transformation parameters), burdened with unidentified outliers had a significant influence on transformation results. Therefore, it was necessary to find a way to automatically identify outliers among reference point coordinates. The JAROCIN network was re-measured and re-adjusted in 2007 in "2000" reference frame. Since the mean error of coordinates was smaller than $2 \mathrm{~cm}$, these coordinates were considered as error free for the purpose of the RANSAC transformation method testing. In the rest of the article this coordinates will be called "catalogue coordinates".

The transformations were performed in three scenarios with different numbers of reference points burdened with gross errors (13, 27 and 45 outliers respectively) and different value of these errors. The artificial errors were added to both $\mathrm{X}$ and $\mathrm{Y}$ coordinates. Number of iterations performed for each scenario with respect to the $\varepsilon$ parameter is presented in Table 1.

Table 1 - Number of RANSAC iterations.

\begin{tabular}{c|c|c|c|c}
\hline Scenario & \multirow{2}{*}{$\begin{array}{c}\text { Number of } \\
\text { outliers }\end{array}$} & $90 \%$ & $95 \%$ & $99.7 \%$ \\
\cline { 3 - 5 } & 13 & 3 & 3 & 7 \\
\hline 1 & 27 & 8 & 10 & 20 \\
\hline 2 & 45 & 81 & 106 & 206 \\
\hline 3 & &
\end{tabular}


The first scenario assumed that $24 \%$ (13 out of 54) of the reference point coordinates included outliers with a magnitude from $0.15-0.30 \mathrm{~m}$. The purpose of the RANSAC algorithm in the process of coordinate transformations is to use only the correct observation. Outliers will not be taken during the estimation of the transformation parameters. The RANSAC algorithm should choose 41 points that are not burdened with gross errors and calculate the correct transformation parameters. Figure 3 and 4 present the histogram of the residuals $\mathrm{X}$ and $\mathrm{Y}$ obtained in the first scenario.

Figure 3 - Histogram of the 41 points $\mathrm{X}$ residual obtained in the first scenario.

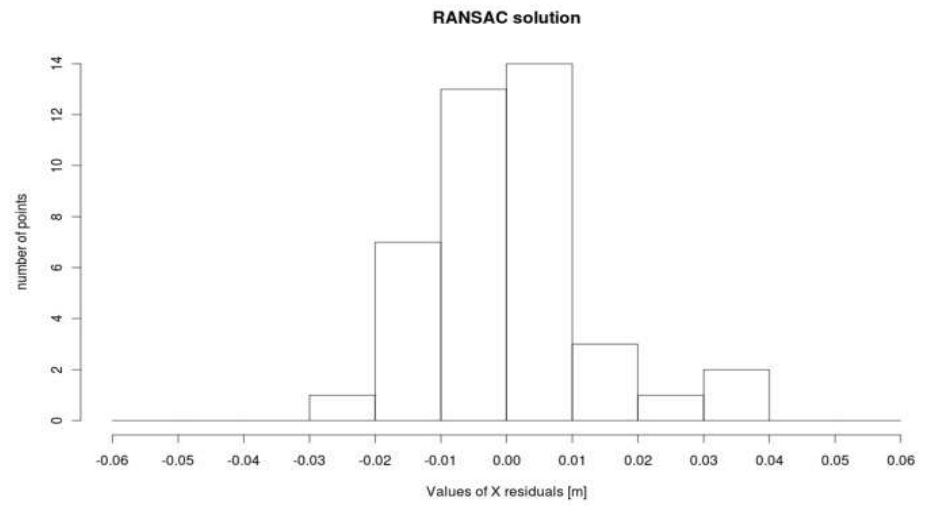

Figure 3 presents a histogram of the $\mathrm{X}$ residuals obtained in the first scenario. The values of these residuals are between -0.03 to $0.04 \mathrm{~m}$.

Figure 4 - Histogram of the 41 points Y residual obtained in the first scenario.

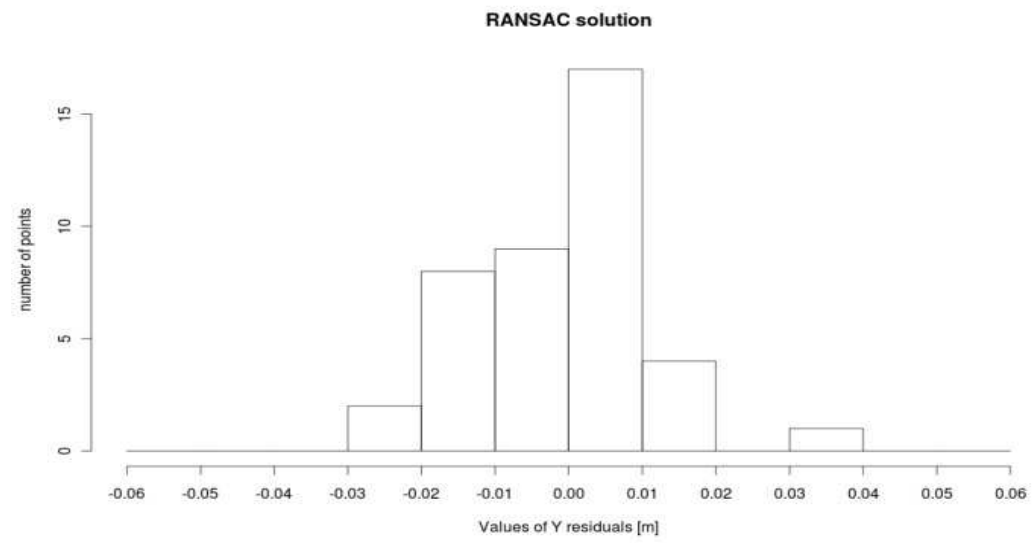

Bol. Ciênc. Geod., sec. Artigos, Curitiba, v. 20, nº 3, p.610-625, jul-set, 2014. 
The Figure 4 shows the histogram of the $\mathrm{Y}$ residuals obtained in the first scenario. In this figure, the values of these residuals are also between -0.03 to 0.04 m.

The RANSAC algorithm identified all outliers and the transformation parameters were computed using 41 correct points. The resulting coordinates obtained from RANSAC transformation were compared with catalogue coordinates and the differences between them were calculated. The resulting coordinate differences are presented as histograms in Figure 5 and 6.

Figure 5 - Histogram of the differences between X coordinates after RANSAC transformation and catalogue values in the first scenario.

\section{RANSAC solution}

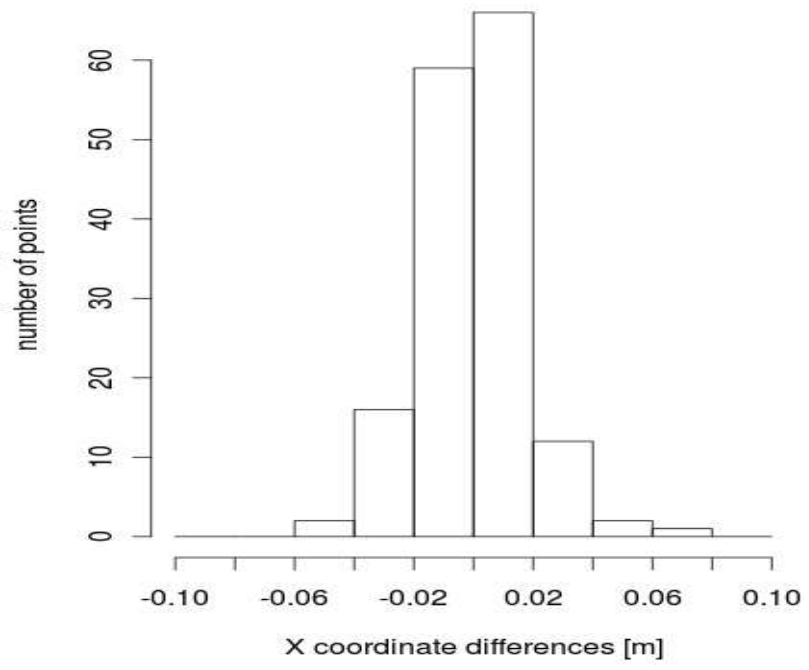

The Figure 5 presents the histogram of the $\mathrm{X}$ coordinate differences obtained in the first scenario. The values of these differences are between -0.06 to $0.08 \mathrm{~m}$. However, more than $80 \%$ of the differences are less then $\pm 0.02 \mathrm{~m}$.

Figure 6 presents the histogram of the $\mathrm{Y}$ coordinate differences obtained in the first scenario. The values of these differences are between -0.04 to $0.04 \mathrm{~m}$.

From Figures 5 and 6 it is clear that all of the coordinate differences are much smaller than the value of gross errors. It shows that the RANSAC algorithm prevented outliers from being used in transformation parameters estimation. 
Figure 6 - Histogram of the differences between Y coordinates after RANSAC transformation and catalogue values in the first scenario.

RANSAC solution

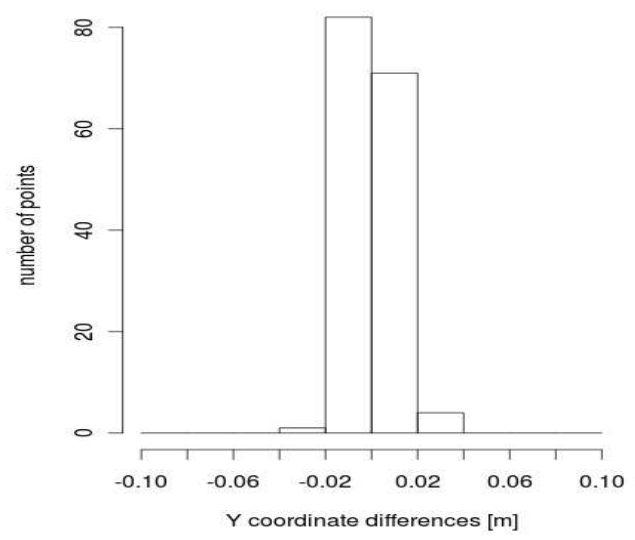

The calculation in the second scenario performed on the same dataset JAROCIN, which includes 158 points and 54 of them are reference points. In this scenario the gross errors of magnitude of 0.15 to $0.30 \mathrm{~m}$ were added to the $\mathrm{X}$ and $\mathrm{Y}$ coordinates of $50 \%$ of reference points (27 out of 54 reference points). The RANSAC algorithm should choose 27 points that are not burdened with gross errors and calculate the correct transformation parameters Then the transformation parameters were estimated and the transformation was performed. The values of the residuals obtained in this scenario are shown in Figures 7 and 8.

Figure 7 - Histogram of the 27 points X residual obtained in the second scenario.

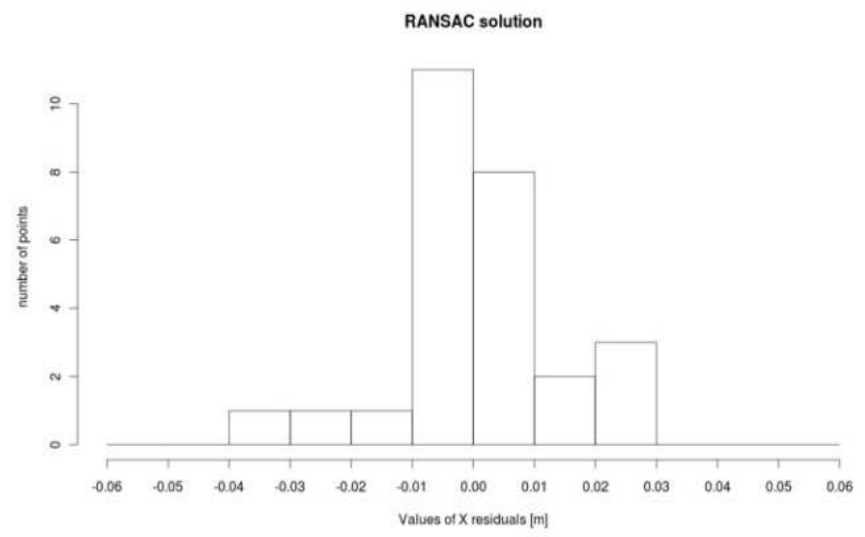

Bol. Ciênc. Geod., sec. Artigos, Curitiba, v. 20, nº 3, p.610-625, jul-set, 2014. 
The Figure 7 presents the histogram of $\mathrm{X}$ residuals obtained in the second scenario. In this scenario, the values of the residuals are between -0.03 and $0.04 \mathrm{~m}$.

Figure 8 - Histogram of the 27 points Y residual obtained in the second scenario.

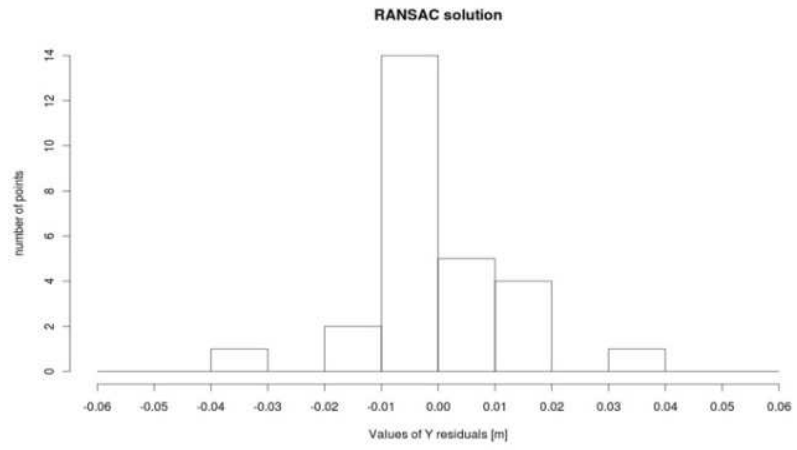

In the Figure 8, the histogram of 27 point residuals is shown. Based on this, it can be determined that more than $90 \%$ of the residuals are less than $+-0.02 \mathrm{~m}$.

In the second scenario the RANSAC algorithm again identified all outliers and the transformation parameters were computed using 27 correct points. Then the transformation of 158 points from local coordinate system to the Polish Coordinate System "2000" was performed. The histograms of the differences between coordinates after transformation and catalogue values are shown in Figures 9 and 10 .

Figure 9 presents the histogram of the $\mathrm{X}$ coordinate differences obtained in the second scenario. The values of these differences are between -0.06 and $0.06 \mathrm{~m}$. However, almost $80 \%$ of the differences are less than $\pm 0.02 \mathrm{~m}$.

Figure 9 - Histogram of the differences between X coordinates after RANSAC transformation and catalogue values in the second scenario.

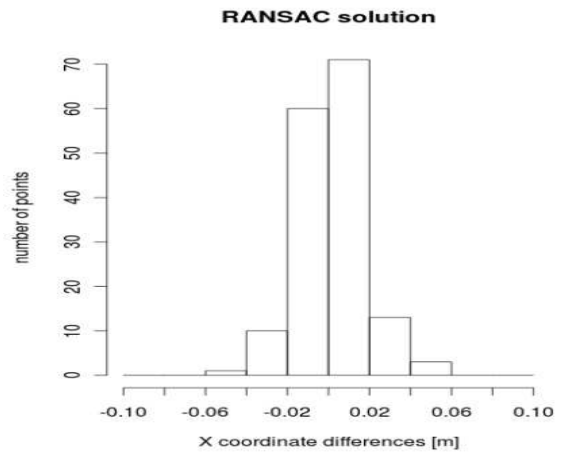

Bol. Ciênc. Geod., sec. Artigos, Curitiba, v. 20, nº 3, p.610-625, jul-set, 2014. 
Figure 10 - Histograms of the differences between coordinates Y after RANSAC transformation and catalogue values in the second scenario.

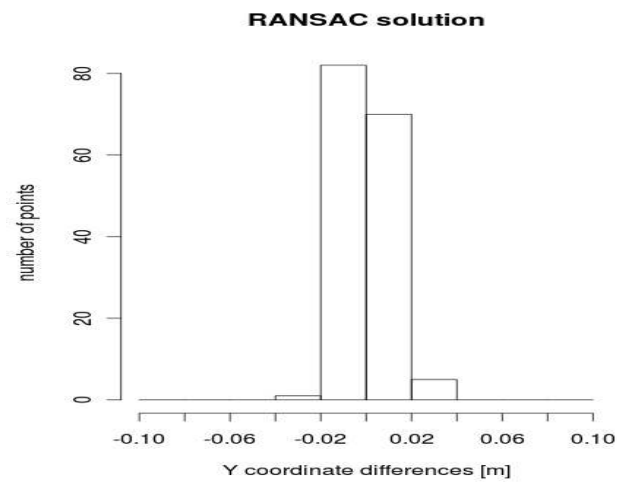

Figure 10 presents the histogram of the $\mathrm{Y}$ coordinate differences obtained in the second scenario. The values of these differences are between -0.04 to $0.04 \mathrm{~m}$ but more than $90 \%$ of the differences are less than $\pm 0.02 \mathrm{~m}$.

From Figures 9 and 10 it is clear that all of the coordinate differences are much smaller than the value of gross errors. It shows that the RANSAC algorithm again prevented outliers from being used in transformation parameters estimation.

The calculation in the third scenario was performed on the same data set JAROCIN. In this scenario the gross errors of magnitude of 0.15 to $0.30 \mathrm{~m}$ were added to the $\mathrm{X}$ and $\mathrm{Y}$ coordinates of $83 \%$ of reference points (45 out of 54 reference points). The RANSAC algorithm should choose 9 points that are not burdened with gross errors and calculate the correct transformation parameters. The residuals, calculated to the nine correct reference point coordinates, are presented in Figures 11 and 12 .

Figure 11 - Histogram of the 9 points $\mathrm{X}$ residual obtained in the third scenario.

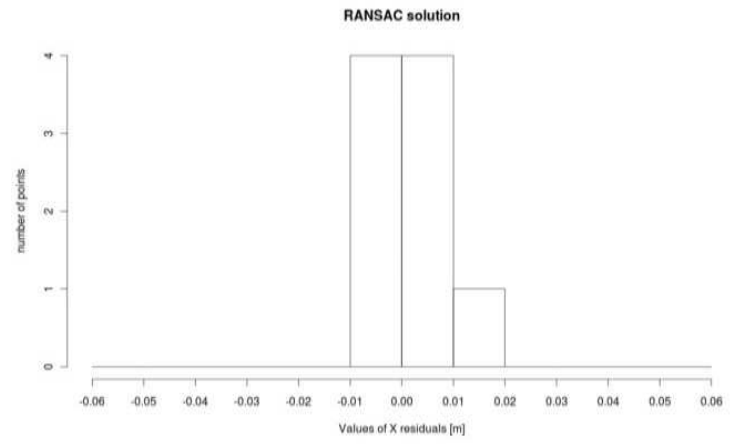

Bol. Ciênc. Geod., sec. Artigos, Curitiba, v. 20, nº 3, p.610-625, jul-set, 2014. 
The Figure 11 presents the histogram of $\mathrm{X}$ residuals obtained in the third scenario. In this scenario, the values of the residuals are between -0.01 and $0.02 \mathrm{~m}$.

Figure 12 - Histogram of the 9 point residuals $\mathrm{Y}$ obtained in the third scenario.

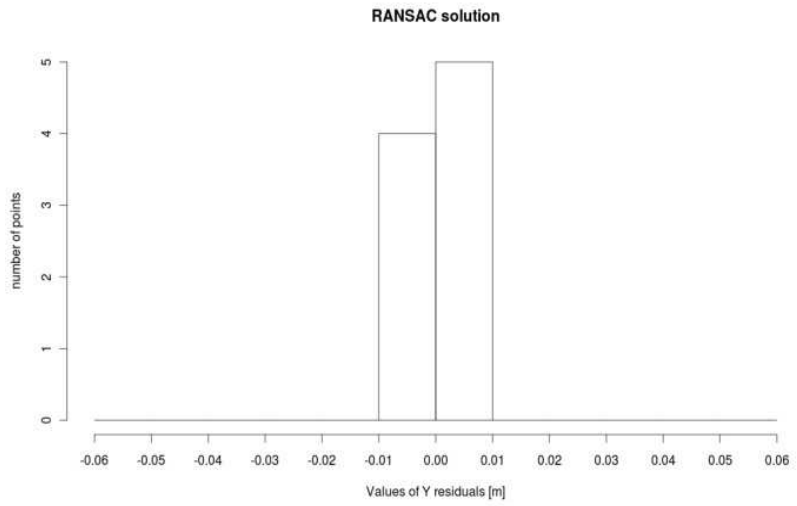

The Figure 12 presents the histogram of $\mathrm{Y}$ residuals obtained in the third scenario. The values of the residuals are about $\pm 0.01 \mathrm{~m}$.

The resulting coordinate differences are presented as histograms in Figures 13 and 14.

Figure 13 - Histogram of the differences between X coordinates after RANSAC transformation and catalogue values in the third scenario.

RANSAC solution

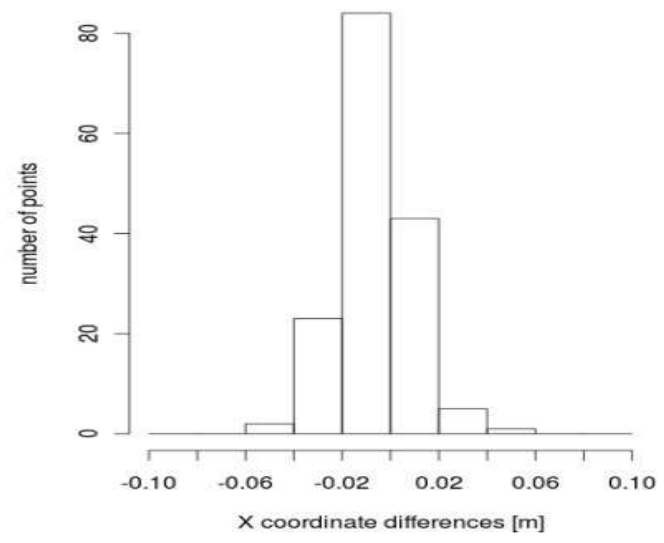

Bol. Ciênc. Geod., sec. Artigos, Curitiba, v. 20, nº 3, p.610-625, jul-set, 2014. 
In the Figure 13, the histogram of the X coordinate differences obtained in the third scenario is presented. The values of these differences are between -0.06 and $0.06 \mathrm{~m}$ as in the second scenario. The results of the transformation are almost the same in those two scenarios.

Figure 14 - Histograms of the differences between Y coordinates after RANSAC transformation and catalogue values in the third scenario.

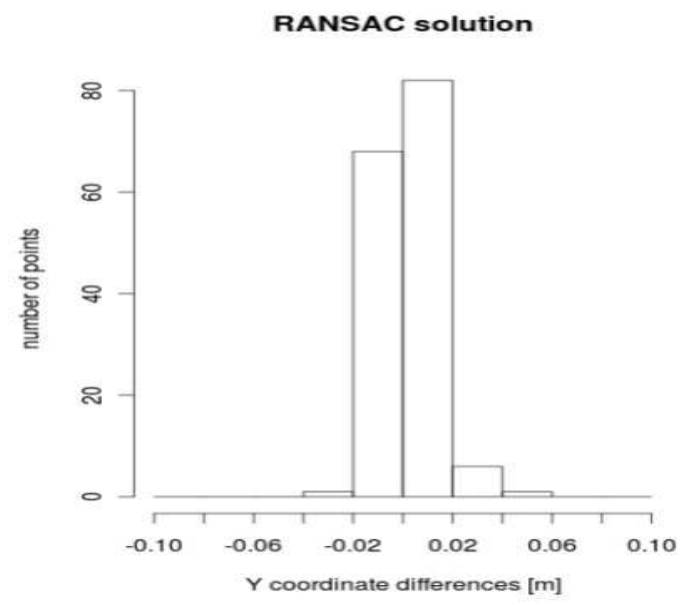

Figure 14 shows the histogram of the $\mathrm{Y}$ coordinate differences obtained in the third scenario. The differences are similar to the $\mathrm{Y}$ coordinate differences in the second scenario and are between -0.04 and $0.06 \mathrm{~m}$.

In this scenario, the coordinates of 45 points are contaminated by outliers. Thus, only 9 out of 54 points are not burdened with gross errors. In this case, traditionally used robust estimation methods would not give the correct result. These methods would recognize those nine correct points as outliers and 45 bad points as correct points. RANSAC transformation correctly identified 9 points (inliers) and the transformation parameters were estimated based on them.

Analyzing the coordinate differences obtained in all scenarios of the calculations, it can be seen that the $\mathrm{X}$ coordinate differences are slightly larger than $Y$ coordinate differences. Since point coordinates used in this experiment were obtained from the GNSS survey (for a second frame) this might be the influence of some surveying errors (due to bad satellite geometry or horizon obstructions at some points). However, it had no effect on the calculation results.

\section{DISCUSSIONS AND CONCLUSIONS}

In order to show the robustness of this approach, the method was applied in three different scenarios, considering different numbers of outliers. It started with a 
case with $24 \%$ of points contaminated with outliers and continued with a $50 \%$ of outliers up to a very high number of $83 \%$ of points contaminated with outliers.

In each variant, the desired results of the calculations were achieved. The algorithm was able to find a correct solution and to eliminate outliers from estimation process. Therefore, the effectiveness of RANSAC algorithm applied to estimation of coordinate transformation parameters is confirmed. The main goal of the study was to confirm the possibility to properly estimate coordinate transformation parameters, when the total number of points burdened with errors is greater than 50\%. Calculations performed in the second and third variants prove the effectiveness of the proposed algorithm, with the number of observations burdened with errors at the level of $83 \%$. In all three scenarios both residuals and calculated distances between catalogue coordinates and coordinates after transformation were at the level of random observation error. The number of required iterations increased with the number of outliers in the data set. To assure for $99.7 \%$ that in the third scenario the algorithm will select two points that are not outliers 206 iterations were required (Table 1).

Despite the advantages of this method, it has some flows. It is not an efficient method from a computational point of view. Number of iterations increases with the number of outliers in the data set and with required probability of successful selection of two inliers. It requires much iterations and many operations which sometimes (especially in the case of large sets) take a longer time than the standard procedure. There is also a risk that the algorithm will not select the proper two points for the best solution (which depends on the a priori selection of $\varepsilon$ parameter). Therefore, an insightful analysis of results is required.

\section{BIBLIOGRAPHICAL REFERENCES}

CHOI, S.; KIM, T.; YU, W. Performance Evaluation of RANSAC Family. Proceedings of the British Machine Vision Conference (BMVC). p. 1-12, 2009.

DUCHNOWSKI, R., Robustness of strategy for testing leveling mark stability based on rank tests. Survey Review. Vol 43(323), pp. 687-699, 2011

FISCHLER, M., BOLLES, R., Random Sample Consensus: A Paradigm for Model Fitting with Applications to Image Analysis and Automated Cartography. Communications of the ACM. Vol 6(24), pp. 381-395, 1981.

GRUBBS, F., Procedures for detecting outlying observations in samples. Technometrics. Vol. 11, No. 1(Feb., 1969), pp. 1-21, 1969

HUBER, P. J., Robust estimation of a location parameter, Annals of Mathematical Statistics, (1), pp. 73-101, 1964

ISACK, H., BOYKOV Y., Energy-based Geometric Multi-Model Fitting. International Journal of Computer Vision. 97(2): 1: 23-147, 2012

JANICKA, J., RAPINSKI, J., M-split transformation of coordinates. Survey Review. Vol. 45, issue 331, pp. 269-274, 2013 
JANICKA, J., Transformation of coordinates with robust estimation and modified Hausbrandt correction. Environmental engineering, (III), pp. 1330-1334, 2011

MURRAY, D.W., TORR, P.H.S., The Development and Comparison of Robust Methods for Estimating the Fundamental Matrix. International Journal of Computer Vision, Vol 24, no 3, pp. 271-300, 1997

WISNIEWSKI, Z., Estimation of parameters in a split functional model of geodetic observations. Journal of Geodesy, (83), pp. 105-120, 2008

(Recebido em abril de 2013. Aceito em abril de 2014). 